\title{
The application of elliptic Fourier analysis in understanding biface shape and symmetry through the British Acheulean
}

Authors: Christian Steven Hoggard ${ }^{1,2}$, John McNabb ${ }^{2}$, and James Nathan Cole ${ }^{3}$

${ }^{1}$ Department of Archaeology and Heritage Studies, School of Culture and Society, Aarhus University, Aarhus, 8000, Denmark.

${ }^{2}$ Department of Archaeology, Faculty of Humanities, University of Southampton, Southampton, SO17 1BJ, United Kingdom.

${ }^{3}$ Department of Geography and Environment, School of Environment and Technology, University of Brighton, Brighton, BN2 4GJ, United Kingdom.

Corresponding author: Christian Steven Hoggard, Department of Archaeology and Heritage Studies, School of Culture and Society, Aarhus University, Aarhus, 8000, Denmark. < C.Hoggard@cas.au.dk > (ORCID: 0000-0002-0022-3605) <+447913352688>

\section{Abstract}

Acheulean biface shape and symmetry has fuelled many discussions on past hominin behaviour in regards to the 'meaning' of biface technology. However, few studies have attempted to quantify and investigate their diachronic relationship using a substantial dataset of Acheulean bifaces. Using the British archaeological record as a case study we first perform elliptic Fourier analysis on biface outlines to quantify and better understand the relationship between biface shape and individual interglacial periods. Using the extracted Fourier coefficients we then detail the nature of symmetry throughout this period, before investigating both shape and symmetry in parallel. The importance of size (through biface length) as a factor in biface shape and symmetry is also considered. Results highlight high levels of symmetry from Marine Isotope Stage (MIS) 13, followed by increasing asymmetry through the British Acheulean. Other observations include a general shift to 'pointed' forms during MIS 9 and 7 and the importance of size in high biface symmetry levels. This article concludes by discussing the potential importance of secondary deposition and palimpsest sites in skewering the observed relationships throughout the Palaeolithic.

Keywords: BIFACE; GEOMETRIC MORPHOMETRICS; ACHEULEAN; SYMMETRY 


\section{Introduction}

For the last sixty years many of the established classificatory frameworks for understanding and interpreting past hunter-gatherer societies have been underpinned by analyses of artefact morphology and the categorisation of morphological variation (Bordes 1961; Roe 1969, 1981; Wymer 1968). For studies in early prehistory, this focus on morphological variation is best exemplified through the numerous debates, analyses, interpretations and reinterpretations of biface variability throughout the Acheulean period (c. $1.7 \mathrm{ma}-200 \mathrm{ka}$ ) (Machin 2009). Given their broad spatial and temporal coverage, ubiquitous to many (but not all) early prehistoric contexts (Lycett and von Cramon-Taubadel 2008), in addition to the clear imposition of intent so early in the archaeological record, it is unsurprising that there is now a significant corpus dedicated to variability and 'meaning' of Acheulean bifaces (Wynn 1995, 2002; Kohn and Mithen 1999; Machin et al. 2007; Machin 2008, 2009; Mithen 2008; Hodgson 2009; Nowell and Chang 2009; Spikins 2012; Lycett 2015; McNabb et al. 2018). It is for these reasons that the biface is now noted as one of the more studied artefact types within early prehistory (Iovita 2010), to the extent that Lycett and colleagues (Lycett et al. 2015: 157) liken the multitude of experimental biface studies to that of the use of fruit flies (Drosophila spp.) as a 'model organism' within the biological sciences. Throughout these studies on biface morphology and 'meaning' two aspects have produced perhaps the greatest interest: aspects of bilateral symmetry and planform shape.

Often noted as a hallmark of cognitive evolution (Wynn 2002; Wynn and Coolidge 2016; $\mathrm{McNabb}$ and Cole 2015), there have been numerous attempts to quantify, interpret and fundamentally understand bilateral symmetry during the Acheulean. Saragusti et al. (1998) represents one of the earliest attempts to quantify biface symmetry throughout early prehistory. Through an analysis of three archaeological contexts in Israel, Saragusti et al. (1998) concluded that symmetry increased over time. There were a number of statistical issues acknowledged by the authors themselves, specifically associated with the analysed sample size. When this dataset was subsequently extended and analysed by Saragusti et al. (2005: 846), it was observed that "the picture emerging is more complex than a simple monotonic increase in the degree of symmetry over time".

An appreciation and understanding of symmetry was also fundamental to the 'sexy handaxe theory' (Kohn and Mithen 1999), which proposed that Darwinian sexual selection accounted (in part) for biface symmetry and thus enabled material culture proxies to act as biological indicators for individual phenotypic fitness. This prompted an extensive discussion on the role of material culture as an extended phenotype (Hodgson 2009; Machin 2008, 2009; Nowell and Chang 2009), best summarised by Spikins (2012).

Following this, biface symmetry was considered by Wynn (2002), who detailed two cognitive 'thresholds', represented through the evidence of Palaeolithic bifaces. The first threshold, categorised by the deliberate imposition of form in the earliest examples of biface technology, were noted as featuring varying levels of bilateral symmetry (with symmetry not always consistent applied). The second threshold, occurring half a million years ago, was defined as the congruence of bilateral planform and cross-section symmetry. Wynn's proposed model prompted a mixture of reactions, with some stressing that biface symmetry was an emerging 
property generated by the extensive flaking of bifaces (Coventry and Clibbens 2002), while others suggested that the recognition of symmetry was an ancient faculty, reflecting the way with which visual stimuli were processed by the brain (Deregowski 2002; Reber 2002). More recently, McNabb et al. (2018:295) note that this discussion led to a view that "... archaeologists should not assume the presence of symmetry in material culture was conscious or culturally learned".

Other researchers have considered the relationship of symmetry to the utilitarian function of the biface. While the use of bifaces in butchery and carcass processing is now extensively attested (Jones 1981; Schick and Toth 1993; Keeley 1980; Mitchell 1996), it was Machin et al. (2007) who first noted in their experimental framework that a large percentage of cutting edge effectiveness could not be solely explained by symmetry (or a number of other linear measurements).

More recently, a number of other arguments and hypotheses have been developed further exploring the 'meaning' of biface symmetry. These include hypotheses of bifaces as advertisements (Machin 2009) and reciprocal altruistic tokens (Spikins 2012). Others have helped to clarify and understand the level of symmetry throughout the Lower and Middle Palaeolithic. For example, Iovita et al. (2017) highlight high levels of symmetry as early as c.700,000 years ago, followed by a longue durée involving the variable imposition of symmetry in different assemblages across time (Hosfield et al. 2018; McNabb et al. 2018). For further details on discussions relating to biface symmetry, see Spikins (2012), Hodgson (2015), McNabb and Cole (2015) and McNabb et al. (2018).

Unsurprisingly, aspects of biface shape and form (size plus shape) are linked to many of the above discussions on biface symmetry e.g. shape as indicators for phenotypic fitness (e.g. Kohn and Mithen 1999), with many of the aforementioned themes or directions of research tackled through a shape-centric perspective. For example, just as biface symmetry has been discussed with reference to butchery efficiency and functionality, so too has biface shape. This includes the recent work by Key and Lycett (2014), who analysed the relationship between biface shape, size (size is here defined as mass) and functionality. Using a large dataset of experimentallyreproduced bifaces, Key and Lycett (2014) demonstrated that biface shape does not have an immediate impact on cutting effectiveness, and that such variation may be related to nonfunctional issues.

In addition, discussions on the role of biface shape have often focused on aspects of biface reduction strategy and changes associated with resharpening (Emery 2010; Iovita 2009; Iovita and McPherron 2011; Li et al. 2015; McPherron 2000; Serwatka 2015; Shipton and Clarkson 2015; White 1998, 2006), and the use of bifaces in understanding underlying social learning mechanisms (Lycett et al. 2015; Schillinger et al. 2015, 2016, 2017). Given the necessity of powerful exploratory and statistical methodologies for cataloguing and understanding biface shape variance, two- and three-dimensional geometric morphometric (GMM) methodologies have been particularly advantageous in this regard (Archer and Braun 2010; Costa 2010; Iovita 2009, 2010; Iovita and McPherron 2011; Key and Lycett 2017; Li et al. 2015; Lycett 2007; Lycett et al. 2006; Schillinger et al. 2017; Shipton and Clarkson 2015; Wang et al. 2012). 
Central to many previous studies on biface shape and symmetry has been an understanding of whether both shape and symmetry become increasingly standardised towards the end of the Acheulean (Saragusti et al. 1998, 2005, Hodgson 2009, 2015; Beyene et al. 2013; Li et al. 2015; McNabb and Cole 2015; McNabb et al. 2018). Despite these studies, there is a distinct absence of exploratory and statistical frameworks which quantify and investigate the diachronic relationship of biface shape and symmetry (independently and concurrently) through the analysis of a large comparative Acheulean dataset. Such studies would be of great benefit to researchers studying broad-scale temporal change in technological behaviour and MIS-specific variability. In addition, such a study would add to the broader literature, challenging and testing the notion of increasing biface shape and symmetry standardisation over time.

Using the British archaeological record as a case study, this paper examines the diachronic relationship in biface shape and symmetry. Specifically, this paper will explore three questions:

1. How does biface shape change throughout the British Acheulean and can increasing standardisation in biface shape be observed?

2. How does biface symmetry change throughout the British Acheulean and can increasing standardisation in biface symmetry be observed?

3. How are the main sources of biface shape variation linked to variations in symmetry, and how does biface size relate to both biface shape and symmetry?

\section{Methodology}

\subsection{Dataset and recording strategy}

To investigate the diachronic relationship of biface shape and symmetry throughout the British Acheulean, and how size is driving any possible correlation, a two-dimensional GMM framework is here presented, encompassing 468 artefacts from ten archaeological sites (Table 1). In their choice, these ten sites represent one of the best-dated and chronostratigraphically secure archaeological sequences in north-western Europe (Hosfield 2011; McNabb 2007). Regarding their raw material, flint is predominant throughout the dataset, with Pontnewydd Cave representing the sole exception, where volcanic raw material was utilised (AldhouseGreen et al. 2012).

In constructing a dataset, the complete number of bifaces from a museum collection were examined. In instances where collections were significantly larger (i.e. deviating from the mean number of bifaces per collection) a random-number generator (https://www.randomizer.org/) was used to sample 50 bifaces from each site. For comparative purposes, the Late Pleistocene site of Lynford (Boismier et al. 2003, 2012), dating to the Marine Isotope Stage (MIS) 4/3 was included. 
Table 1. The British Lower and Middle Palaeolithic assemblages used for this study (total: 468); BM: British Museum, United Kingdom; NMW: National Museum of Wales, United Kingdom; NAU: Northampton Archaeological Unit, United Kingdom.

Despite the prevalence of three-dimensional GMM methodologies in recent years (Archer et al. 2015, 2017; Herzlinger et al. 2017), a two-dimensional methodology analysing digital photographs of bifaces is here presented. While greater artefact coverage benefits the analytical power of an analysis, when used with caution, the examination of two-dimensional photographs serves a number of advantages, including the potential to record and analyse considerably larger datasets, including archival data, illustrations, and open-access repositories.

Digital photographs of each biface were captured by JM and JNC, with the dataset expanded through the Marshall et al. (2002) database, curated by the Archaeological Data Service (ADS) (http://archaeologydataservice.ac.uk/archives/view/bifaces/). To minimise lens distortion (distortion through perspective and optics), a suitable recording strategy was implemented, with profile corrections performed in the CorelDraw Graphics Suite (X7).

In order to analyse biface shape, an outline was first created in the CorelDraw Graphics Suite (using the 'Trace Outline' function), with the original photograph subsequently deleted to reduce pixel noise. The outline was set to a thickness of one pixel and screened for breaks and errors. Incomplete curves were subsequently closed, with all changes also standardised to one pixel in thickness. These outlines were then exported as a Portable Network Graphics (.png file extension) for subsequent recording and analysis.

\subsection{Elliptic Fourier Analysis}

To investigate biface shape and symmetry, the outlines were examined through elliptic Fourier analysis (EFA), a common method of closed outline shape analysis, extending on from the Fourier series first derived by Jean Baptiste Joseph Fourier (1768-1830). Through EFA, an outline is transformed into an infinite series of repeating trigonometric functions (or harmonics), with four Fourier coefficients per harmonic retained (Ferson et al. 1985; Giardina and Kuhl 1977; Kuhl and Giardina 1982). Through biological studies by Iwata et al. (1998), and later by Iwata et al. (2002) and Yoshioka et al. (2004), the four derived coefficients from the $n^{\text {th }}$ harmonic can be classified into two categories, pertaining to symmetrical $\left(\mathrm{a}_{n}\right.$ and $\left.\mathrm{d}_{n}\right)$ and asymmetrical $\left(\mathrm{b}_{n}\right.$ and $\mathrm{c}_{n}$ ) variations in two-dimensional shape. As a closed outline is constructed by the ratio of these coefficients, an index of symmetry can be calculated from the ratio of symmetric harmonic coefficients to the sum of the absolute value of all harmonic coefficients ( 1.0 being calculated as complete bilateral symmetry). Central to the quantification and analysis of biface shape, through the above procedure, is a robust protocol for evaluating rotation. In orienting the bifaces, outlines were rotated for maximum symmetry through the elliptic best-fitting procedure of the first harmonic. The authors do acknowledge that error may be incorporated in planform siding, as non-corresponding edges may be analysed. And as 
legacy data (i.e. photographs) are here utilised, previous planform siding techniques focusing on scar density or 'doming' (Shipton and Clarkson 2015; Lycett et al. 2015) would prove difficult to determine. However, as studies highlight that the main sources of shape variation largely reflect symmetric changes in shape (Archer et al. 2015, 2017; Shipton and Clarkson 2015; Lycett et al. 2015), and as the first two major sources of variation (i.e. principal component scores) will be examined in detail, this error will be minimal.

In comparison to other closed outline methods including coordinate-point eigenshape (MacLeod 1999), Fourier radius variation and Fourier tangent angles (Zahn and Roskies 1972), and the fitting of polynomial curves (Rogers and Fog 1989), EFA boasts a number of methodological advantages. For this study, EFA permits the quantitative assessment of both biface symmetry and shape through the same analysis, in comparison to the above techniques. EFA also does not require data points to be of the same number or evenly spaced, and thus allows the analysis of complex two-dimensional edges. Furthermore, through the automation of outlines and subsequent digitisation, a replicable methodology with minimal subjectivity and inter-observer error (e.g. Hardaker and Dunn 2005; Underhill 2007) is achieved.

In order to analyse outlines through EFA, the .png files were first synthesised into one thinplate spline (.tps) file in tpsUtil v.1.69 (Rohlf 2017a), with Cartesian coordinates and positions for each image created using the 'Outline object' tool in tpsDig2 v.2.27 (Rohlf 2017b). Both software programs are open-source and are available online (http://life.bio.sunysb.edu/ee/rohlf/software.html). As these outlines do not require the same number of landmarks throughout the .tps file, and in order to capture as much of the original shape as possible, the raw outline was stored. In total, the 468 bifaces feature $1808 \pm 829$ Cartesian coordinates. The .tps file was then imported into Momocs v. 1.16 (Bonhomme et al. 2014) for the R Environment (R Deverlopment Core Team 2017), with an associated .csv file detailing the biface site, size (through biface length in millimetres) and MIS. In standardising the outlines prior to the EFA, all outlines were normalised to a common centroid $(0,0)$, and rescaled using their centroid size following guidelines by Bonhomme et al. (2017). Normalisation through rotation was unnecessary as this is achieved through elliptic fitting of the first harmonic. In choosing a sufficient number of harmonics necessary to capture sufficient biface shape, the 'calibrate_harmonicpower_efourier' and 'calibrate_deviations_efourier' functions in Momocs were used (and supported through the 'calibrate_reconstructions_efourier' function). Through this procedure 13 harmonic powers were necessary for $99 \%$ harmonic power, here defined as capturing sufficient biface shape. For more information on the fundamentals of EFA and EFA coefficients see Caple et al. (2017).

\subsection{Analytical and exploratory procedure}

To address the first research question, the main sources of shape variation within the ten sites were explored through a principal component analysis (EFA-PCA). The contributions for each principal component were examined through a scree plot, with shape transformations for each major principal component documented. A visual examination of the five interglacials, and their spatial configuration to the theoretical shape changes (i.e. the principal component 
scores), was conducted through confidence ellipses (66.66\%). To explore if bifaces become increasingly standardised through the British Acheulean, variance in the first two principal components were examined through visual examination of the EFA-PCA plot and through boxand-whisker plots (Tukey style).

In exploring if specific biface shapes can be linked to particular periods, and if differentiation in biface shapes can be observed across the five interglacials, a discriminant analysis (with leave-one-out cross-validations), following guidelines by Kovarovic et al. (2011), was conducted. To perform the discriminant analysis, $99 \%$ cumulative shape variance totalling the first twenty-one principal component axes were retained. A discriminant analysis for the individual sites was not performed as three sites (Elveden, Bowman's Lodge and Pontnewydd Cave) do not meet the suggested sample size values (Klecka 1980; Kovarovic et al. 2011; McGarigal et al. 2000).

To support the exploratory exercise, the five interglacials were examined within a statistical multivariate framework. Specifically, a multivariate analysis of variance (MANOVA) of 99\% cumulative shape variance was performed within their respective MIS. A null hypothesis $\left(\mathrm{H}_{0}\right)$ of same populations for each variable was assumed, with statistical significance here defined at the $1 \%$ significance level (i.e. $\alpha=0.01$ ). Bonferroni-corrected $\mathrm{p}$ values are used throughout this procedure.

In addressing the second research question, the calculated symmetry values were first examined through visual comparison of individual archaeological sites and their respective MIS through box-and-whisker plots. These plots are then supported with calculated coefficient of variation (CV) values for each MIS. Together, these two methods should indicate if increasing standardisation in biface symmetry is observed. To examine if the interglacials have different distributions in symmetry values, a Shapiro-Wilk normality test was first performed for each MIS, with a suitable test for significance then conducted. As four groups feature non-normal distributions, a Kruskal-Wallis test was performed, followed by pairwise Wilcoxon ranked sum tests.

Finally, in addressing the third research question, and the underlying relationship between biface shape, size and symmetry, the first five sources of shape variance were first examined in relation to the calculated symmetry values through Pearson's product-moment correlation and visual examination of the scatterplots. The length measurements were then examined against the calculated symmetry values, and the major principal components, also through Pearson's product-moment correlation and visual examination of the produced scatterplots.

Through this exploratory and analytical framework, diachronic changes in biface shape and symmetry were examined, with the underlying influence of biface size determined.

All graphics and analyses within this text were produced with the help of Momocs v.1.2.9 (Bonhomme et al. 2014), tidyverse v.1.2.1 (Wickham 2009) and cowplot v.0.9.3 (https://github.com/wilkelab/cowplot) for the R environment ( $\mathrm{R}$ Development Core Team 2017). In encouraging greater data transparency, guidelines from Marwick (2017) were undertaken to ensure that analyses are replicable. We therefore include the tps file, the 
necessary metadata (in .csv format), and the R script used for this article. A copy of all the necessary files can also be found on the Open Science Framework (https://osf.io/td92j/).

\section{Results}

When the outlines are examined through EFA-PCA, the first two principal components (theoretical sources of shape variation) account for $78.75 \%$ cumulative variance, with the first 21 principal components accounting for $99 \%$ cumulative shape variance. In their transformation, the first principal component reflects shape changes from narrow and morepointed biface shapes to rounded and more-circular biface shapes, with the second principal component extending from narrow-based biface shapes to triangular biface shapes. The third principal component (7.74\% cumulative shape variance) represents the first shape change based on asymmetric differences (see the PCContrib function in the R script for more information).

With respect to how these components manifest differences in shape over time, the principal component plot (Figure 1) demonstrates considerable difference between certain interglacial periods, in their clustering and spatial configuration. Visual inspection of the EFA-PCA highlights considerable overlap in the shape distribution (among the first two principal axes) of examples dating from MIS 9 and MIS 7 (with more positive PC1 and PC2 values), and similarity between the earliest examples of bifaces within the dataset (MIS 13), and examples from Lynford (MIS 4/3). In their totality, on the basis of the first two components, there is increasing shape variance over each period until MIS 4/3.

Through further examination of principal component scores, for the first two axes (Figures 2a and 2c), similarities between bifaces in MIS 9 and MIS 7, and examples in MIS 13 and MIS $4 / 3$ are further highlighted. In both examples, MIS 13 bifaces have the least variation in PC1 and PC2 scores, exemplifying a more standardised shape. When examined on an individual site level (Figure $2 \mathrm{~b}$ and $2 \mathrm{~d}$ ), the high degree of shape standardisation at Boxgrove, and to a lesser extent at Warren Hill, can be observed.

Figure 1. An exploration of biface shape and Marine Isotope Stage (MIS) through an elliptic Fourier principal component analysis (EFA-PCA). Confidence ellipses are here set to two-thirds $(66.66 \%)$.

Figure 2. An exploration of the first two principal component scores through box-andwhisker plots (Tukey style) for both period (Figure 2a and Figure 2c) and individual sites (Figure 2b and Figure 2d). Site order correlates with Marine Isotope Stage (MIS) and does not reflect a strict chronological order. 
Through an assessment of the first 21 principal components ( $99 \%$ cumulative shape variance), a MANOVA was performed to test for statistical significance between the different interglacial periods and their respective shape variance. Through this, statistical significance below the designated 1\% significance threshold was observed (Hotelling-Lawley: 0.5847, F: 3.0733 p: < 0.0001). When examined further through Hotelling T tests (Table 2), statistical significance to the designated threshold was observed between all possible combinations demonstrating differences in the shape variance of all interglacial periods. Interestingly, when the first 21 principal components are examined through a discrimination analysis (with leave-one-out cross-validation), only $36.11 \%$ of all bifaces (169/468) could be correctly classified to their respective MIS. In their totality, the MANOVA and discriminant analysis demonstrate that while each interglacial period features statistically significant group variance, with distinct trends in specific shapes for each period, degrees of overlap indicate that absolute periodspecific shapes cannot be inferred. For more information on both the MANOVA and discriminant analysis (for each MIS and site), refer to the R script.

Table 2. A MANOVA pairwise table (with Bonferroni-corrected $p$ values) of $99 \%$ cumulative shape variance (first 21 principal components) tested with respect to Marine Isotope Stage (MIS); significance codes: less than $0.0001={ }^{6 * * *}$; less than $0.001={ }^{6 * *}$; less than $0.01={ }^{*} *$; greater than $0.01={ }^{\prime}$,

In examining biface symmetry, calculated as the AD harmonic coefficients divided by their amplitude, a unimodal asymmetric distribution with moderate skewness is observed for all examples, with the majority of bifaces centred on roughly 0.95 (95\%) symmetry (Figure 3a). In better understanding what this score and distribution refers to, see Figure 4.

When symmetry investigated through their respective interglacial period (Figure 3b), examples in MIS 13 and MIS 4/3 feature higher median symmetry values than the collective median. Interestingly, throughout the intermediate periods, greater variation in the quartile range and low median symmetry values for each MIS are observed. On an individual site level (Figure $3 c$ ), the temporal trend in increasing asymmetry until MIS $4 / 3$ is again noted, with high levels of symmetry noted at Broom, Boxgrove, Warren Hill and Lynford, and greater variation and thus greater asymmetry in MIS 7 examples. Descriptive statistics of the calculated symmetry values (Table 3 ) further support a trend in the British Acheulean of increasing asymmetry until the end of the Middle Palaeolithic, with decreased symmetry means, lower minimum symmetry values and higher standard deviations and CVs for MIS 11, MIS 9 and MIS 7.

A non-parametric Kruskal-Wallis rank-sum test demonstrated that statistical significance was observed when biface symmetry values are assessed in relation to MIS (chi-squared: 63.104, $d f: 4, p:<0.0001$. In further detail, a Wilcoxon rank sum test (Table 4) demonstrates that MIS 13 cannot be differentiated from MIS 4/3 biface symmetry levels but can be differentiated from the intermediate interglacial periods. 
Figure 3. An examination of symmetry (AD harmonic coefficients/amplitude) through a histogram (Figure 3a), and two box-and-whisker plots (Tukey style) examining symmetry against individual Marine Isotope Stage (Figure 3b), and against individual sites (Figure 3c).

Figure 4. Three shapes depicting the variation in symmetry throughout the dataset (as calculated through the AD harmonic coefficients divided by their amplitude).

Table 3. Descriptive statistics of symmetry values for each MIS (sample size in subscript)

Table 4. A non-parametric pairwise Wilcoxon rank sum test (with Bonferroni-corrected $p$ values) for symmetry values and with respect to Marine Isotope Stage (MIS); significance codes: less than $0.0001=$ '***'; less than $0.001=$ '**'; less than $0.01=$ '*'; greater than $0.01=$ '?

Through Pearson's product-moment correlation of the main sources of shape variance and calculated symmetry values, no linear relationship between shape and symmetry for the first two principal components can be observed. For later principal components (Figure 5), extreme values are associated with greater asymmetry. In their totality the correlation-based analyses demonstrate that the main sources of biface shape variation throughout the British Acheulean are reflected in symmetric shape changes.

Finally, to understand the underlying factor of size in the shape and symmetry of British Acheulean bifaces, product-moment correlations were performed (Figure 6). A productmoment correlation of size and symmetry reveals statistical significance to the 0.01 alpha level, specifically that larger bifaces often feature higher symmetry levels ( $\mathrm{t}: 3.28$, df $=466$, p: 0.0011, slope: 0.1502). Furthermore, the first two major sources of shape variation (the extension from rounded to pointed shapes, and from narrow-based to triangular shapes) were both found to be statistically significant.

Figure 5. Scatterplots (with smoothed conditional means) for the five principal components assessed against symmetry (AD harmonic coefficients/amplitude)

Figure 6. Scatterplots (with smoothed conditional means) for size and symmetry (A) and the three major sources of shape variation (B-D)

\section{Discussion}


This paper performed a two-dimensional GMM framework on a large biface dataset to quantify and investigate biface symmetry and shape through the British Acheulean. Specifically, this paper aimed at examining whether biface shape and symmetry became increasingly standardised through time, with the late Middle Palaeolithic site of Lynford acting as a point of comparison. Questions of specific biface shape linked to particular MIS periods, and the role of size in biface shape and symmetry were also explored.

The analytical framework chosen highlighted considerably high levels of symmetry in MIS 13, reinforcing previous studies examining Acheulean symmetry (McNabb and Cole 2015; Iovita et al. 2017; McNabb et al. 2018; White and Foulds 2018), followed by increasing asymmetry. The recorded variation in symmetry, equivalent to Boxgrove, does not appear (through the above sites) until the late Middle Palaeolithic. A similar pattern is noted for shape variance, with greater standardisation in biface shape documented in MIS 13 and again in MIS 4/3; while decreasing shape standardisation was noted in MIS 11, in MIS 9 and MIS 7. Despite these diachronic changes, the statistical differences between the respective interglacial periods through discriminant analyses, demonstrate that individual shapes are poor indicators of particular MIS periods. Further investigations highlighted that the main sources of shape variation are associated with changes in symmetry, and that specific shapes are associated with different sizes (Figure 6).

An analysis of biface shape highlights similarities in the main sources of shape variance to that noted in other studies which have examined biface datasets through a GMM framework (Iovita and McPherron 2011; Serwatka 2015). In this, it is perhaps beneficial for future studies to develop a grammar, and a language of categorising bifaces, based on the observed changes in GMM analyses, and away from traditional shape-based typologies (e.g. Wymer 1968).

Unexpectedly, our analysis also highlights an observation first noted in the linear morphometric analyses by Roe (1969), and recently developed by Bridgland and White (2015; White and Bridgland 2018), of biface shape trends and their pertinence as potential cultural markers. While specific shapes could not be attributed to distinct MIS periods of the Acheulean, the analysis highlighted differences in MIS-specific shape variation, with a preference for more pointed biface shapes from MIS 9 onwards. Exceptions to this trend do exist, the more positive PC scores indicate more rounded examples at Broom for example, however this study further highlights the appropriateness of long-standing biface classificatory schemes (see Bridgland and White 2018 for more information).

Investigations into the nature of shape and symmetry in each interglacial period may allude to the importance of on-site accumulation and the time-depth of each archaeological site. In instances of higher symmetry and shape standardisation, for example Boxgrove and Lynford, horizons are in-situ and are thought to represent strict contemporaneous events, representing a few generations at maximum (Boismier et al. 2012; Roberts and Parfitt 1999). In contrast, many archaeological sites with high asymmetry and shape variance seem to relate more to secondary context sites and palimpsests, representing the accumulation of artefacts over thousands or even tens of thousands of years (McNabb 2007; McNabb and Cole 2015). While site-formation processes can only be proxies for accumulation, and durations cannot be credibly estimated, one must not rule out the influence of deposition in understanding potential biface variability, 
a point also highlighted by Moncel et al. (2015). Crucially, if reworking from higher/older deposits can be eliminated, a palimpsest in this context can be of advantage as it samples a range of potential variability across a given time period (for example the duration over which a river terrace accumulates). Further work is necessary to better understand the role of this potentially important factor and the overall impact on how researchers interpret site assemblages.

This study exemplifies the interpretive potential of large biface datasets through an exploratory and analytical GMM framework, and supports previous interpretations (e.g. Saragusti et al. 2005, Cole 2015, McNabb et al. 2018, White and Foulds 2018) using an independent methodology, that symmetry does not seem to be consistently present through the Acheulean. The key to identifying diachronic changes in symmetry is to identify trends in the shift in the medians and interquartile ranges of assemblages constrained by tight temporal frameworks such as in the British Middle Pleistocene. The importance of the interpretative frameworks (White and Bridgland 2018, Foulds and White 2018) currently being applied to the British sequence is that they provide a behavioural explanation for the increase in diversity (handaxe shape and symmetry) seen in the British late Middle Pleistocene.

Through the integration of other early biface sites and a more nuanced understanding of site accumulation and its relationship with hominin occupation and behaviour, the Acheulean can be better quantified and understood. This in turn provides a platform for testing many of the earlier publications discussing the behavioural 'meaning' of these morphological attributes.

\section{Conclusion}

Through an exploratory and analytical GMM analysis of a large biface dataset it has been possible to examine the nature of diachronic change in biface shape and symmetry through the British Acheulean record. A number of observations were documented, supporting previous views on a high level of shape and symmetry standardisation in MIS 13 and on the inconsistent application of symmetry across the Acheulean time range. This work has also highlighted the variability in symmetry that is present within the British Acheulean record, and alludes to the potential roles of occupation duration, assemblage accumulation, and even demography in understanding that variability. However, in understanding the true 'meaning' of biface shape and symmetry, the integration of much larger datasets from mainland Europe, Asia and Africa is now necessary.

\section{Acknowledgments}

The authors would like to thank Vincent Bonhomme for our exchanges on EFA and the Momocs package, Cory Stade for proofreading the manuscript, and the five anonymous reviewers for their helpful comments. The authors would particularly like to thank Shannon McPherron for his comments on this paper. Any errors that remain are those of the authors. CSH is supported by the Independent Research Fund Denmark (grant \#6107-00059B). 


\section{Conflict of Interests}

On behalf of all authors, the corresponding author states that there is no conflict of interest.

\section{References}

Aldhouse-Green, S., Peterson, R. \& Walker, E. (2012). Neanderthals in Wales: Pontnewydd and the Elwy Valley Caves. Oxbow Books, Oxford..

Archer, W., \& Braun, D. R. (2010). Variability in bifacial technology at Elandsfontein, Western cape, South Africa: a geometric morphometric approach. Journal of Archaeological Science, 37(1), 201-209.

Archer, W., Gunz, P., Van Niekerk, K. L., Henshilwood, C. S., \& McPherron, S. P. (2015). Diachronic change within the Still Bay at Blombos Cave, South Africa. PLOS ONE, 10(7).

Archer, W., Pop, C. M., Rezek, Z., Schlager, S., Lin, S. C., Weiss, M., et al. (2017). A geometric morphometric relationship predicts stone flake shape and size variability. Archaeological and Anthropological Sciences, 1-13.

Beyene, Y., Katoh, S., WoldeGabriel, G., Hart, W. K., Uto, K., Sudo, M., et al. (2013). The characteristics and chronology of the earliest Acheulean at Konso, Ethiopia. Proceedings of the National Academy of Sciences.

Boismier, W. A., Gamble, C., \& Coward, F. (2012). Neanderthals among mammoths: excavations at Lynford Quarry, Norfolk. English Heritage.

Boismier, W., Schreve, D. C., White, M. J., Robertson, D. A., Stuart, A. J., Etienne, S., et al. (2003). A Middle Palaeolithic site at Lynford Quarry, Mundford, Norfolk : interim statement. Proceedings Of The Prehistoric Society, 69, 315-324. Bonhomme, V., Forster, E., Wallace, M., Stillman, E., Charles, M., \& Jones, G. (2017). Identification of inter- and intra-species variation in cereal grains through geometric morphometric analysis, and its resilience under experimental charring. Journal of Archaeological Science, 86, 60-67.

Bonhomme, V., Picq, S., Gaucherel, C., \& Claude, J. (2014). Momocs: Outline Analysis Using R. Journal of Statistical Software, 56, 1-24.

Bordes, F. (1961). Mousterian Cultures in France. Science, 134(3482), 803-810.

Bridgland, D. R., \& White, M. J. (2015). Chronological variations in handaxes: patterns detected from fluvial archives in north-west Europe. Journal of Quaternary Science, $30(7), 623-638$.

Caple, J., Byrd, J., \& Stephan, C. N. (2017). Elliptical Fourier analysis: fundamentals, applications, and value for forensic anthropology. International Journal of Legal Medicine, 131(6), 1675-1690.

Costa, A. G. (2010). A geometric morphometric assessment of plan shape in bone and stone acheulean bifaces from the Middle Pleistocene site of Castel di Guido, Latium, Italy. In New Perspectives on Old Stones: Analytical Approaches to Paleolithic Technologies 
(pp. 23-41).

Coventry, K. R., \& Clibbens, J. (2002). Does Complex Behaviour Imply Complex Cognitive Abilities? Behavioral and Brain Sciences, 25(3), 406. doi:10.1017/S0140525X02250074

Deregowski, J. B. (2002). Is symmetry of stone tools merely an epiphenomenon or similarity? Behavioral and Brain Sciences, 25(3), 406-407. doi:10.1017/S0140525X02260070

Emery, K. (2010). A re-examination of variability in handaxe form in the British Palaeolithic. Archaeology. PhD Dissertation. University College London.

Ferson, S., Rohlf, F. J., \& Koehn, R. K. (1985). Measuring Shape Variation of TwoDimensional Outlines. Systematic Zoology, 34(1), 59-68.

Giardina, C., \& Kuhl, F. (1977). Accuracy of Curve Approximation by Harmonically Related Vectors with Elliptical Loci. Computer Graphics and Image Processing, 6, 277-285.

Hardaker, T., \& Dunn, S. (2005). The flip test - A new statistical measure for quantifying symmetry in stone tools. Antiquity, https://www.antiquity.ac.uk/projgall/hardaker.

Herzlinger, G., Goren-Inbar, N., \& Grosman, L. (2017). A new method for 3D geometric morphometric shape analysis: The case study of handaxe knapping skill. Journal of Archaeological Science: Reports, 14, 163-173.

Hodgson, D. (2009). Symmetry and humans: Reply to Mithen's "Sexy handaxe theory." Antiquity, 83(319), 195-198.

Hodgson, D. (2015). The symmetry of Acheulean handaxes and cognitive evolution. Journal of Archaeological Science: Reports, 2, 204-208.

Hosfield, R. (2011). The british lower palaeolithic of the early middle pleistocene. Quaternary Science Reviews, 30(11-12), 1486-1510.

Hosfield, R., Cole, J., \& McNabb, J. (2018). Less of a bird's song than a hard rock ensemble. Evolutionary Anthropology, 27(1), 9-20.

Iovita, R. (2010). Comparing Stone Tool Resharpening Trajectories with the Aid of Elliptical Fourier Analysis. In S. Lycett \& P. Chauan (Eds.), New Perspectives on Old Stones (pp. 235-253). New York, NY: Springer New York.

Iovita, R. (2009). Ontogenetic scaling and lithic systematics: method and application. Journal of Archaeological Science, 36(7), 1447-1457.

Iovita, R. (2010). Comparing stone tool resharpening trajectories with the aid of elliptical fourier analysis. In New Perspectives on Old Stones: Analytical Approaches to Paleolithic Technologies (pp. 235-253).

Iovita, R., \& McPherron, S. P. (2011). The handaxe reloaded: A morphometric reassessment of Acheulian and Middle Paleolithic handaxes. Journal of Human Evolution, 61(1), 6174.

Iovita, R., Tuvi-Arad, I., Moncel, M. H., Desprieae, J., Voinchet, P., \& Bahain, J. J. (2017). High handaxe symmetry at the beginning of the European Acheulian: The data from la Noira (France) in context. PLoS ONE, 12(5), 1-25.

Iwata, H., Nesumi, H., Ninomiya, S., Takano, Y., \& Ukai, Y. (2002). Diallel Analysis of Leaf 
Shape Variations of Citrus Varieties Based on Elliptic Fourier Descriptors. Breeding Science, 52, 89-94.

Iwata, H., Niikura, S., Matssura, S., Takano, Y., \& Ukail, Y. (1998). Evaluation of variation of root shape of Japanese radish ( Raphanus sativus L .) based on image analysis using elliptic Fourier descriptors. Euphytica.

Jones, P. R. (1981). Experimental Implement Manufacture and Use; A Case Study from Olduvai Gorge, Tanzania. Philosophical Transactions of the Royal Society of London. Series B, Biological Sciences (1934-1990), 292(1057), 189-195.

Shick, K.D., \& Toth, N. (1993). Making Silent Stones Speak. Weidenfeld and Nicolson, London.

Keeley, L. H. (1980). Experimental Determination of Stone Tool Uses. University of Chicago Press.

Key, A. J. M., \& Lycett, S. J. (2017). Reassessing the production of handaxes versus flakes from a functional perspective. Archaeological and Anthropological Sciences, 9(5), 737 753.

Klecka, W. (1980). Discriminant Analysis. Advances in Neural Information Processing Systems, 17(60), 1569-1576.

Kohn, M., \& Mithen, S. (1999). Handaxes: Products of sexual selection? Antiquity, 73(281), $518-526$.

Kovarovic, K., Aiello, L. C., Cardini, A., \& Lockwood, C. A. (2011). Discriminant function analyses in archaeology: Are classification rates too good to be true? Journal of Archaeological Science, 38(11), 3006-3018.

Kuhl, F. P., \& Giardina, C. R. (1982). Elliptic Fourier features of a closed contour. Computer Graphics and Image Processing, 18(3), 236-258.

Li, H., Kuman, K., \& Li, C. (2015). Quantifying the reduction intensity of handaxes with 3D technology: A pilot study on handaxes in the danjiangkou reservoir region, central China. PLoS ONE, 10(9).

Lycett, S. J. (2007). Is the Soanian techno-complex a Mode 1 or Mode 3 phenomenon? A morphometric assessment. Journal of Archaeological Science, 34(9), 1434-1440.

Lycett, S. J. (2015). Cultural evolutionary approaches to artifact variation over time and space: Basis, progress, and prospects. Journal of Archaeological Science, 56, 21-31.

Lycett, S. J., Schillinger, K., Kempe, M., \& Mesoudi, A. (2015). Learning in the Acheulean: Experimental insights using handaxe form as a "model organism" In Learning Strategies and Cultural Evolution During the Palaeolithic (pp. 155-166).

Lycett, S. J., \& von Cramon-Taubadel, N. (2008). Acheulean variability and hominin dispersals: a model-bound approach. Journal of Archaeological Science, 35(3), 553562.

Lycett, S. J., von Cramon-taubadel, N., \& Eren, M. I. (2016). Levallois: Potential Implications for Learning and Cultural Transmission Capacities. Lithic Technology, 41(1), 1-20.

Lycett, S. J., von Cramon-Taubadel, N., \& Foley, R. A. (2006). A crossbeam co-ordinate 
caliper for the morphometric analysis of lithic nuclei: A description, test and empirical examples of application. Journal of Archaeological Science, 33(6), 847-861.

Machin, A. (2009). The role of the individual agent in Acheulean biface variability: A multifactorial model. Journal of Social Archaeology, 9(1), 35-58.

Machin, A. J. (2008). Why handaxes just aren't that sexy: a response to Kohn \& Mithen (1999) Darwinian demography and sexual dimorphism. Antiquity, 82(317), 761-766.

Machin, A. J., Hosfield, R. T., \& Mithen, S. J. (2007). Why are some handaxes symmetrical? Testing the influence of handaxe morphology on butchery effectiveness. Journal of Archaeological Science, 34(6), 883-893.

MacLeod, N. (1999). Generalizing and Extending the Eigenshape Method of Shape Space Visualization and Analysis. Paleobiology, 25(1), 107-138.

Marwick, B. (2017). Computational Reproducibility in Archaeological Research: Basic Principles and a Case Study of Their Implementation. Journal of Archaeological Method and Theory, 24(2), 424-450.

McGarigal, K., Cushman, S., \& Stafford, S. (2000). Multivariate Statistics for Wildlife and Ecology Research. Analysis (Vol. Springer-V).

McNabb, J. (2007). The British lower Palaeolithic: Stones in contention. The Br. Lower Palaeolithic: Stones in Contention.

McNabb, J., \& Cole, J. (2015). The mirror cracked: Symmetry and refinement in the Acheulean handaxe. Journal of Archaeological Science: Reports, 3, 100-111.

McNabb, J., Cole, J., \& Hoggard, C. S. (2018). From side to side: Symmetry in handaxes in the British Lower and Middle Palaeolithic. Journal of Archaeological Science: Reports, 17.

McPherron, S. P. (2000). Handaxes as a measure of the mental capabilities of early hominids. Journal of Archaeological Science, 27(8), 655-663.

Mitchell, J. C. (1996). Studying Biface Utilisation at Boxgrove: Roe Deer Butchery with Replica Handaxes. Lithics, 16, 64-69.

Mithen, S. J. (2008). "Whatever turns you on": A response to Anna Machin, "Why handaxes just aren't that sexy." Antiquity, 82 (317), 766-769..

Moncel, M-H., Ashton, N., Lamotte, A., Tuffreau, A., Cliquet, D. \& Despriée. (2015). The Early Acheulian of north-western Europe. Journal of Anthropological Archaeology, 40, 302-331.

Nowell, A., \& Chang, M. L. (2009). The Case Against Sexual Selection as an Explanation of Handaxe Morphology. PaleoAnthropology, 77-88.

R Development Core Team. (2014). R: A language and environment for statistical computing. R: A Language and Environment for Statistical Computing, 55, 275-286. http://www.r-project.org/.

Reber, R. (2002). Reasons for the preference for symmetry. Behavioral and Brain Sciences, 25(3), 415-416.

Roberts, M. B., \& Parfitt, S. A. (1999). Boxgrove. A Middle Pleistocene hominid site at 
Eartham Quarry, Boxgrove, West Sussex.

Roe, D. A. (1969). British Lower and Middle Palaeolithic Handaxe Groups. Proceedings of the Prehistoric Society, 34, 1-82.

Roe, D. A. (1981). The Lower and Middle Palaeolithic Periods in Britain. London: Routledge \& Kegan Paul.

Rogers, D. F., \& Fog, N. R. (1989). Constrained B-spline curve and surface fitting. Computer-Aided Design, 21(10), 641-648.

Rohlf, F. J. (2017a). tpsUtil v.1.69. State University of New York at Stony Brook.

Rohlf, F. J. (2017b). tpsDig2 v.2.27. State University of New York at Stony Brook.

Saragusti, I., Karasik, A., Sharon, I., \& Smilansky, U. (2005). Quantitative analysis of shape attributes based on contours and section profiles in artifact analysis. Journal of Archaeological Science, 32(6), 841-853.

Saragusti, I., Sharon, I., Katzenelson, O., \& Avnir, D. (1998). Quantitative analysis of the symmetry of artefacts: Lower Paleolithic handaxes. Journal of Archaeological Science, 25(8), 817-825.

Schillinger, K., Mesoudi, A., \& Lycett, S. J. (2015). The impact of imitative versus emulative learning mechanisms on artifactual variation: Implications for the evolution of material culture. Evolution and Human Behavior, 36(6), 446-455.

Schillinger, K., Mesoudi, A., \& Lycett, S. J. (2016). Copying error, evolution, and phylogenetic signal in artifactual traditions: An experimental approach using "model artifacts." Journal of Archaeological Science, 70, 23-34.

Schillinger, K., Mesoudi, A., \& Lycett, S. J. (2017). Differences in Manufacturing Traditions and Assemblage-Level Patterns: the Origins of Cultural Differences in Archaeological Data. Journal of Archaeological Method and Theory, 24(2), 640-658.

Serwatka, K. (2015). Bifaces in plain sight: testing elliptical Fourier analysis in identifying reduction effects on Late Middle Palaeolithic bifacial tools. Litikum, 3, 13-25.

Shipton, C., \& Clarkson, C. (2015). Handaxe reduction and its influence on shape: An experimental test and archaeological case study. Journal of Archaeological Science: Reports, 3, 408-419.

Spikins, P. (2012). Goodwill hunting? Debates over the "meaning" of Lower Palaeolithic handaxe form revisited. World Archaeology, 44(3), 378-392.

Underhill, D. (2007). Subjectivity inherent in by-eye symmetry judgements and the large cutting tools at the Cave of Hearths, Limpopo Province, South Africa. Papers from the Institute of Archaeology, 18(2005), 101-113.

Wang, W., Lycett, S. J., von Cramon-Taubadel, N., Jin, J. J. H., \& Bae, C. J. (2012). Comparison of handaxes from Bose Basin (china) and the western Acheulean indicates convergence of form, not cognitive differences. PLOS ONE, 7(4).

White, M. J. (1998). On the Significance of Acheulean Biface Variability in Southern Britain. Proceedings of the Prehistoric Society, 64, 15-44.

White, M. J. (2006). Axeing cleavers: reflections on broad-tipped large cutting tools in the 
British earlier Paleolithic. In N. Goren-Inbar \& G. Sharon (Eds.), Axe Age: Acheulian Tool-making from Quarry to Discard (pp. 365-386).

White, M. J. and Bridgland, D. R. (2018). Thresholds in lithic technology and human behaviour in MIS 9 Britain. In: M. Pope, J. McNabb \& C. Gamble (Eds.) Crossing the Human Threshold: Dynamic Transformation and Persistent Places during the Middle Pleistocene. Routledge (pp. 165-192).

White, M. J. and Foulds, F. (2018). Symmetry is its own reward: on the character and significance of Acheulean handaxe symmetry in the Middle Pleistocene. Antiquity, 92(362), 304-319.

Wickham, H. (2009). Ggplot2. Elegant Graphics for Data Analysis. doi:10.1007/978-0-38798141-3

Wymer, J. (1968). Lower Palaeolithic Archaeology in Britain: As Represented by the Thames Valley. John Baker.

Wynn, T. (1995). Handaxe enigmas. World Archaeology, 27(1), 10-24.

Wynn, T. (2002). Archaeology and cognitive evolution. Behav Brain Sci, 25(3), 338-389.

Wynn, T., \& Coolidge, F. L. (2016). Archeological insights into hominin cognitive evolution. Evolutionary anthropology, 25(4), 200-213.

Zahn, C. T., \& Roskies, R. Z. (1972). Fourier descriptors for plane closed curves. IEEE Transactions on Computers, C-21(3), 269-281. 